\title{
CORRECTION
}

Open Access

\section{Correction to: Peripheral immune system in aging and Alzheimer's disease}

Wei Cao ${ }^{*}$ and Hui Zheng ${ }^{*}$

\section{Correction}

The original article [1] contained several small omissions and errata contained in Tables 2 and 3; these errors have now been corrected.

Received: 8 October 2018 Accepted: 8 October 2018

Published online: 24 October 2018

\section{Reference}

1. Cao W, Zheng H. Peripheral immune system in aging and Alzheimer's disease. Mol Neurodegener. 2018;13:51 https://doi.org/10.1186/s13024-018-0284-2.

\footnotetext{
*Correspondence: wei.cao@bcm.edu; huiz@bcm.edu Huffington Center on Aging, Houston, TX 77030, USA 